\title{
Solitary Schwannoma of the Cecum: Case Report and Review of the Literature
}

\author{
José Wilson Benevides de Mesquita Neto ${ }^{a}$ \\ Renato Mazon Lima Verde Leal ${ }^{\mathrm{a}} \quad$ Eurivaldo Valente de Brito ${ }^{\mathrm{b}}$ \\ Diego Fonseca Cordeiro $^{b}$ Marcelo Leite Vieira Costa ${ }^{a}$ \\ ${ }^{a}$ Department of Gastrointestinal Oncology, Institute of Cancer of Ceara, and \\ ${ }^{\mathrm{b}}$ School of Medicine, State University of Ceara, Fortaleza, Brazil
}

\section{Key Words}

Gastrointestinal tract $\cdot$ Schwannoma $\cdot$ Cecum $\cdot$ S-100 protein

\begin{abstract}
A 78-year-old woman presented with an abdominal mass diagnosed by ultrasound and computed tomography. The patient underwent a laparotomy, during which a retroperitoneal tumor adherent to the cecum wall was identified. Microscopically, it showed spindle-cell proliferation in whorls, with low mitotic count ( 2 per 50 high-power fields) and was strongly positive for S-100 protein and vimentin. The final diagnosis was benign schwannoma of the cecum and no further treatment was required. Large intestine schwannomas are extremely rare tumors and only a few cases of schwannoma of the cecum have been reported to date.
\end{abstract}

\section{Introduction}

Schwannomas are benign slow-growing mesenchymal neoplasms that arise from Schwann cells with a low malignant potential. They are rare tumors, occurring most commonly intracranially in acoustic nerves or spinal nerves. Gastrointestinal schwannoma (GIS) represents only $2-6 \%$ of all submucosal tumors of the gastrointestinal tract [1], with the stomach being the most affected organ (60-70\%) [2]. The incidence of GIS in the cecum, because of the rare occurrence, is not reported in the literature.

GIS is a distinctive neoplasm usually difficult to distinguish from others tumors of the gastrointestinal wall, such as gastrointestinal stromal tumors (GIST), lipomas, leiomyomas and leiomyossarcomas [2], despite the fact that GIS has a well-defined immunohistochemical 
pattern that is different from the others tumors, showing positive S-100 protein and vimentin in tumor cells [3].

Here, we present a very rare case of schwannoma of the cecum and review the literature, focusing on the different aspects of diagnosis and treatment of this tumor.

\section{Case Report}

A 79-year-old woman presented to her physician with recurrent pain in the right upper abdomen following meals, associated with nausea and vomiting. She had also complained of hyporexia with a weight loss of 11 pounds over the prior 3 months. The patient had no history of previous lower abdominal pain, melena, hematochezia or surgical interventions. An abdominal ultrasound was ordered, which detected gallbladder stones and also a hyperechoic mass in right iliac fossa. Further investigation with abdominal computed tomography (CT) showed a well-defined round mass of $10 \mathrm{~cm}$ in maximal diameter behind the cecum, with homogenous and minimal contrast enhancement (fig. 1).

The patient underwent a laparotomy. The intraoperative findings were a retroperitoneal mass with no cleavage plane with the cecum wall (fig. 2), and cholelitiasis. A frozen section of the retroperitoneal specimen was suggestive of GIST. Right colectomy with no lymph node dissection was performed, followed by cholecystectomy.

Macroscopic examination of the resected segment showed a solid cystic lesion in the cecum wall, which measured $9.0 \times 7.5 \mathrm{~cm}$, with minimal margin of $3 \mathrm{~cm}$. Light microscopic examination of the specimen showed a mesenchymal neoplasm of the cecum wall, with spindle cells and mild atypia arranged in whorls, and a low mitotic count $(2$ per 50 highpower fields). The diagnosis was a low-grade mesenchymal spindle-cell neoplasm, and further investigation with immunohistochemical staining (IHS) was necessary for the differential diagnosis. IHS was strongly positive for vimentin and S-100 protein and negative for HMB-45, CD-34, CD-117, compatible with schwannoma.

The patient was discharged 7 days after the surgery and no further treatment was indicated in follow-up.

\section{Discussion}

Schwannoma is a benign neurogenic neoplasm that originates from Schwann cells; it is slow growing with low malignant potential. It usually affects the peripheral nerves of limbs, the spinal cord and central nervous system [1]. Primary and isolated GIS are not frequent, and occur most commonly in the stomach and jejunum, with the cecum rarely affected. Because of the lack of uniformity in terminology of neurogenic tumors (schwannoma, neurilemmoma, neurofibroma, plexiform neurofibromatosis), their incidence in the large bowel is not known [4], and only a few case have been reported to date.

The symptoms depend on tumor size, although the disease can be totally asymptomatic, presenting as an incidental finding in imaging exams. Nevertheless, it can cause fecal occult bleeding, intussusceptions and bowel obstruction [3]. The preoperative diagnosis of these tumors is usually difficult. CT and magnetic resonance imaging can establish the tumor site, its characteristics and relationships with other organs [2]. However, the final diagnosis can only be confirmed by histopathological examination. The light microscopy with hematoxylin and eosin stain is not accurate to distinguish the neurogenic from the myogenic spindle-cell tumors, and IHS with broad-panel markers is mandatory for this differential diagnosis. 
Schwannomas are neurogenic tumors and usually show S-100 protein and vimentin, while they are negative for desmin, keratin, glial fibrillary acidic protein, smooth muscle and muscle-specific actin, CD34 and EMA 1 [5]. In this case, IHS was strongly positive for S-100 protein and vimentin. GIS reported in the literature is associated with peritumoral lymphoid reaction. Intracellular PAS-positive crystalloids could be a marker of cellular differentiation of Schwann cells [3].

The intraoperative frozen section analysis can help to diagnose the main tumor lineage, and eventually it can suggest the malignant behavior of the neoplasm. This method could help the surgeon during the operation. The treatment for schwannoma is surgery with free margins [6]. The prognosis of patients with schwannoma of the cecum is good after an adequate surgical resection, and no adjuvant therapy is necessary. Recurrence or metastasis in a long follow-up is rare [1].

\section{References}

-1 Inagawa S, Hori M, Shimazaki J, Matsumoto S, Ishii H, Itabashi M, Adachi S, Kawamoto T, Fukao K: Solitary schwannoma of the colon: report of two cases. Surg Today 2001;31:833-838.

$\checkmark 2$ Levy AD, Quiles AM, Miettinen M, Sobin LH: Gastrointestinal schwannomas: CT features with clinicopathologic correlation. AJR 2005;184:797-802.

-3 Tomozawa S, Masaki T, Matsuda K, Yokoyama T, Ishida T, Muto T: A schwannoma of the cecum: a case report and review of Japanese schwannomas in the large intestine. J Gastroenterol 1998;33:872-875.

-4 Miettinen M, Shekitka KM, Sobin LH: Schwannomas in the colon and rectum. A clinicopathologic and immunohistochemical study of 20 cases. Am J Surg Pathol 2001;25:846-855.

-5 Nakayama T, Yoshizaki A, Naito S, Wen CY, Alipov G, Yakata Y, Sekine I: Expression of Ets-1 protooncoprotein in gastrointestinal stromal tumors, leiomyomas and schwannomas. World J Gastroenterol 2006;12:1743-1746.

6 Daimaru Y, Kido H, Hashimoto H, Enjoji M: Benign schwannoma of the gastrointestinal tract: a clinicopathologic immunohistochemical study. Hum Pathol 1988;19:257-264.

J.W.B. Mesquita Neto and M.L.V. Costa contributed equally to this work. 


\section{Case Reports in Oncology}

\begin{tabular}{l|l}
\hline Case Rep Oncol 2013;6:62-65 \\
\hline DOI: $10.1159 / 000346785$ & $\begin{array}{l}\text { ○ 2013 S. Karger AG, Basel } \\
\text { www.karger.com/cro }\end{array}$ \\
\hline
\end{tabular}

de Mesquita Neto et al:: Solitary Schwannoma of the Cecum: Case Report and Review of the Literature

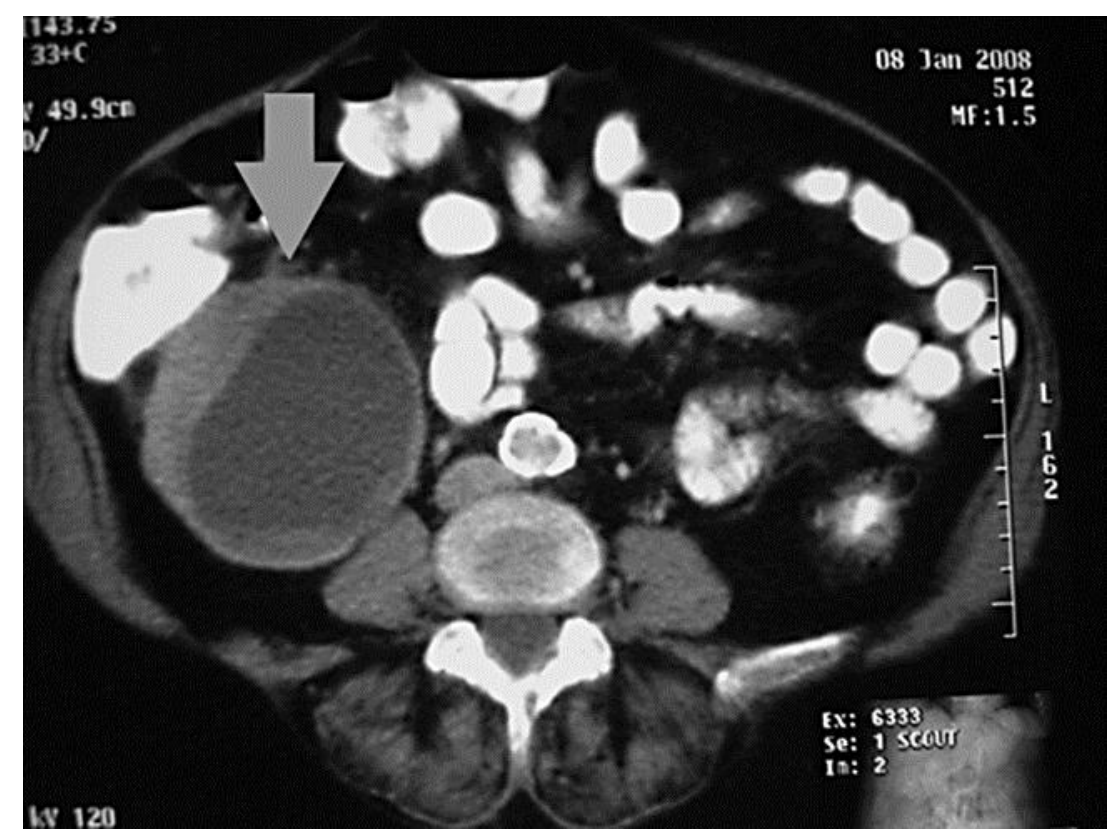

Fig. 1. Abdominal CT showing a retroperitoneal mass (arrow) dorsal to the cecum.

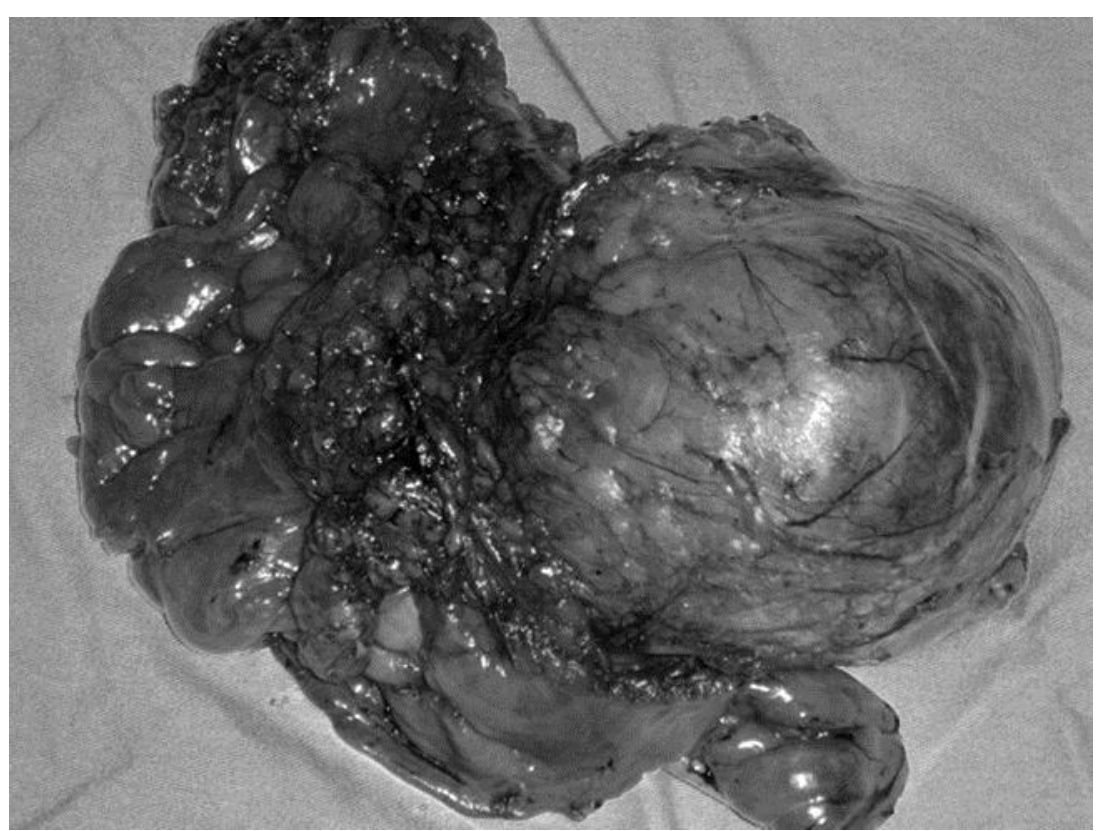

Fig. 2. Product of en bloc resection of the cecum adherent to the retroperitoneal tumor and right colon. 